\title{
Diamond-like carbon
}

\author{
J Robertson \\ Engineering Dept, Cambridge University, Cambridge CB2 1PZ, UK
}

\begin{abstract}
Diamond-like carbon (DLC) is a dense, partially $\mathrm{sp}^{3}$ bonded form of amorphous carbon prepared by ion beam or plasma deposition and frequently used as a hard coating material. Its $\mathrm{sp}^{3}$ bonding arises from $\mathrm{C}^{+}$ions penetrating surface layers and giving a quenched-in density increase. The formation of DLC can be viewed as a phase transition to a denser metastable phase. The atomic structure of DLC consists of a network of $\mathrm{sp}^{3}$ and $\mathrm{sp}^{2}$ sites. The $\pi$ states of $\mathrm{sp}^{2}$ sites control the electronic properties and the connectivity of $\mathrm{sp}^{3}$ sites controls the mechanical properties.
\end{abstract}

\section{Introduction}

There is great current interest in hard ceramics prepared by vapour-deposited such as diamond, diamond-like carbon (DLC), cubic BN, boron carbides and TiN. Diamond's unique properties make it an excellent coating material and a potentially important high temperature semiconductor $[1,2]$. While the technology of diamond deposition develops, interest turned to related materials such as DLC [1,3-5]. DLC is a dense, metastable form of amorphous carbon $(\mathrm{a}-\mathrm{C})$ or hydrogenated amorphous carbon $(\mathrm{a}-\mathrm{C}: \mathrm{H})$ containing a significant $\mathrm{sp}^{3}$ bonding. The $\mathrm{sp}^{3}$ bonding confers valuable 'diamond-like' properties such as mechanical hardness, low friction, optical transparency and chemical inertness. Although DLC films have poorer properties than diamond films, they have some advantages, notably deposition at room temperature, deposition onto Fe or plastic substrates and superior surface smoothness.

\section{Deposition}

Diamond itself can be deposited by various chemical vapour deposition (CVD) methods such as hot filament, microwave plasma, etc [1,2]. Hydrocarbon source gases are used and the role of the power source is to create an excess of atomic hydrogen which alters the thermodynamic stability of the depositing surface. DLC was originally prepared by ion beam deposition [6] and is now prepared by many methods such as magnetron sputtering [7], ion sputtering [8], laser plasma deposition [9], plasma deposition [4] and ion plating [5]. The common factor in these process is deposition from a beam containing medium energy (10$500 \mathrm{eV}$ ) ions [1]. This links DLC to the wider field of ion-beam modification of materials [10].

Plasma deposition can be used to prepare CVD diamond, a-C:H and the important semiconductor a-Si:H so it is of interest to compare the preferred conditions for each case. Diamond is typically grown on substrates held at $700-900^{\circ} \mathrm{C}$ from a $99: 1 \mathrm{H}_{2} /$ methane gas mixture under moderate pressures $(250 \mathrm{~Pa})$ in microwave plasmas, to maximise the atomic hydrogen flux and minimise the ion bombardment of the film. The major growth species are radicals such as $\mathrm{CH}_{3}{ }^{\prime}$. Diamond-like a-C: $\mathrm{H}$ is grown on room temperature substrates from pure hydrocarbons such as acetylene, methane or benzene, under conditions of low pressure ( $3 \mathrm{~Pa}$ ), moderate $\mathrm{RF}$ power, low pressure ( $3 \mathrm{~Pa}$ ) and negative substrate bias to maximise the positive ion flux at the substrate [4]. High quality a-Si: $\mathrm{H}$ is grown from pure silane at low RF or microwave power and medium pressure onto substrates held at $250^{\circ} \mathrm{C}$ [11]. These conditions give a growth species of $\mathrm{SiH}_{3}$, radicals, moderate surface mobility, and minimum ion bombardment of the sample. 


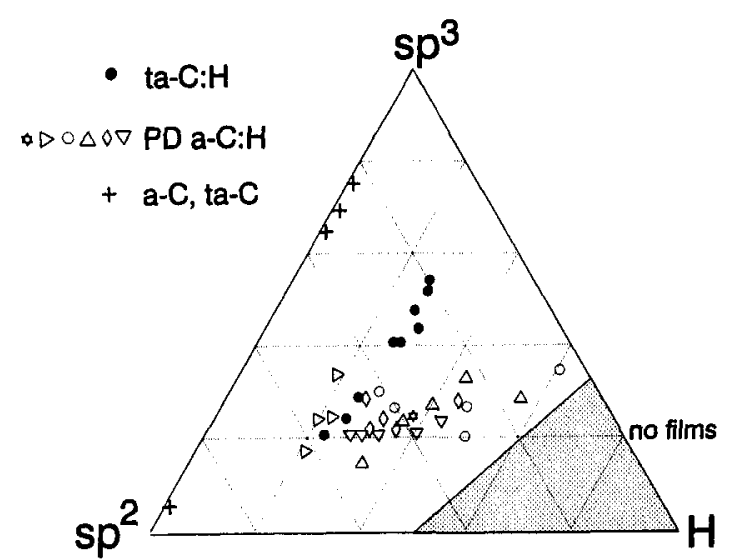

Fig. 1. Ternary diagram showing $\mathrm{sp}^{3}, \mathrm{sp}^{2}$ and $\mathrm{H}$ content of a-C, PD a-C:H [12] and ta-C:H [20].

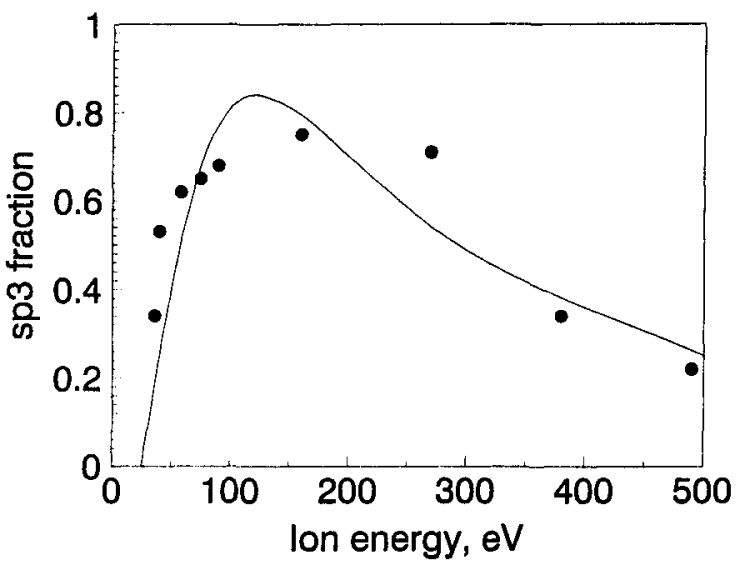

Fig. 2. $s p^{3}$ fraction vs. ion energy for ta-C deposited from filtered ion beam [16].

\section{Properties of DLC}

The nature of the DLC depends on the deposition process used. The $\mathrm{sp}^{3}$ and $\mathrm{H}$ content is most conveniently displayed on a ternary phase diagram [12] as in Fig. 1. The three corners correspond to diamond, graphite and the hydrocarbons, respectively. There is an excluded region at high $\mathrm{H}$ contents, where molecular solids cannot form. The familiar forms of non-crystalline carbon such as glassy carbon and evaporated a-C lie in the $\mathrm{sp}^{2}$ corner. Sputtering methods produce hard but predominantly $\mathrm{sp}^{2}$ bonded a-C [7]. Ion-assisted sputtering onto well-cooled substrates can produce a highly sp ${ }^{3}$ bonded a-C [8]. Laser plasma methods can produce rather highly $\mathrm{sp}^{3}$ bonded a-C films [9].

A particularly useful form of ion beam deposition is filtered ion beam deposition in which a magnetic filter removes neutrals, particulates and other ions and allows deposition from a monochromatic, single species ion beam [13-16]. The resulting a-C attains an $\mathrm{sp}^{3}$ content of up to $85 \%$ and is called highly tetrahedral a-C or 'ta-C' [15]. Diffraction data suggest that its atomic structure is truly amorphous and resembles the 4-fold coordinated random network of a-Si, except for the finite content of 3-fold sites [17]. The properties of ta-C depend strongly on the energy of the ion beam. The $\mathrm{sp}^{3}$ content, density and hardness each pass through a maximum at an optimum ion energy of order $140 \mathrm{eV}$ [16](Fig. 2). Ta-C films also possess a very high intrinsic compressive stress arising from the deposition process which turns out to be a key signature of DLC [15]. A linear correlation is found between $\mathrm{sp}^{3}$ fraction and density (Fig. 3) and the stress (Fig. 4).

Plasma deposition (PD) is the most popular means of producing a-C:H [4]. The substrate is attached to the RF powered electrode which acquires a negative DC self-bias, so the substrate attracts positive ions from the plasma. The proportion of ions in the total deposition flux is relatively low, typically $10 \%$ [18]. The

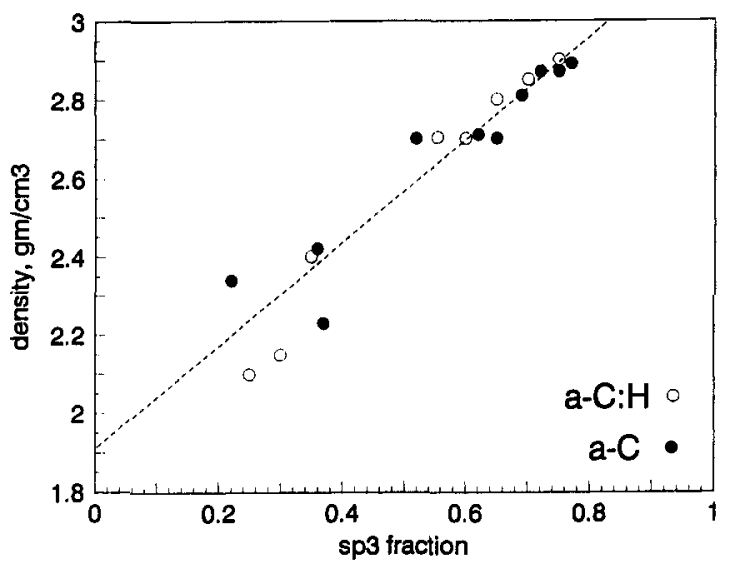

Fig. 3. Density vs. $\mathrm{sp}^{3}$ content for ta-C [16] and ta-C:H [20].

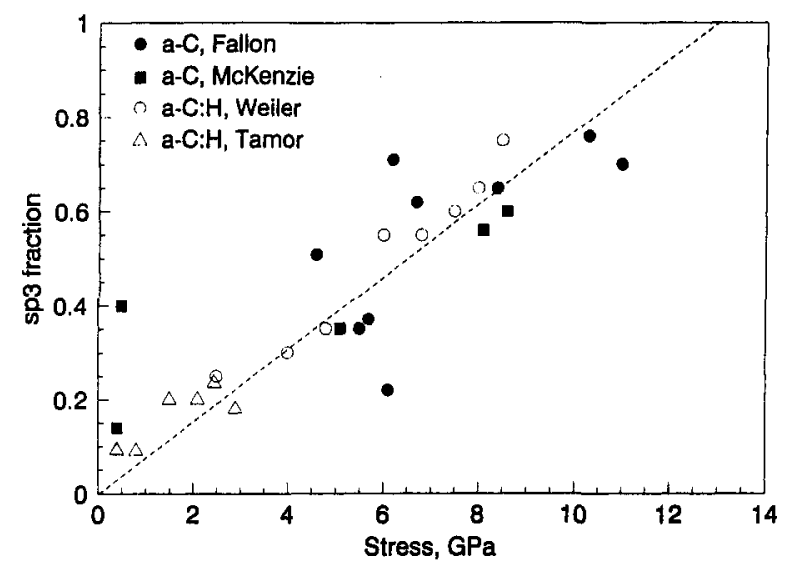

Fig. 4. Compressive stress vs. sp3 fraction for ta-C $[15,16], \mathrm{PD}$ a-C:H [19] and ta-C:H [20]. 


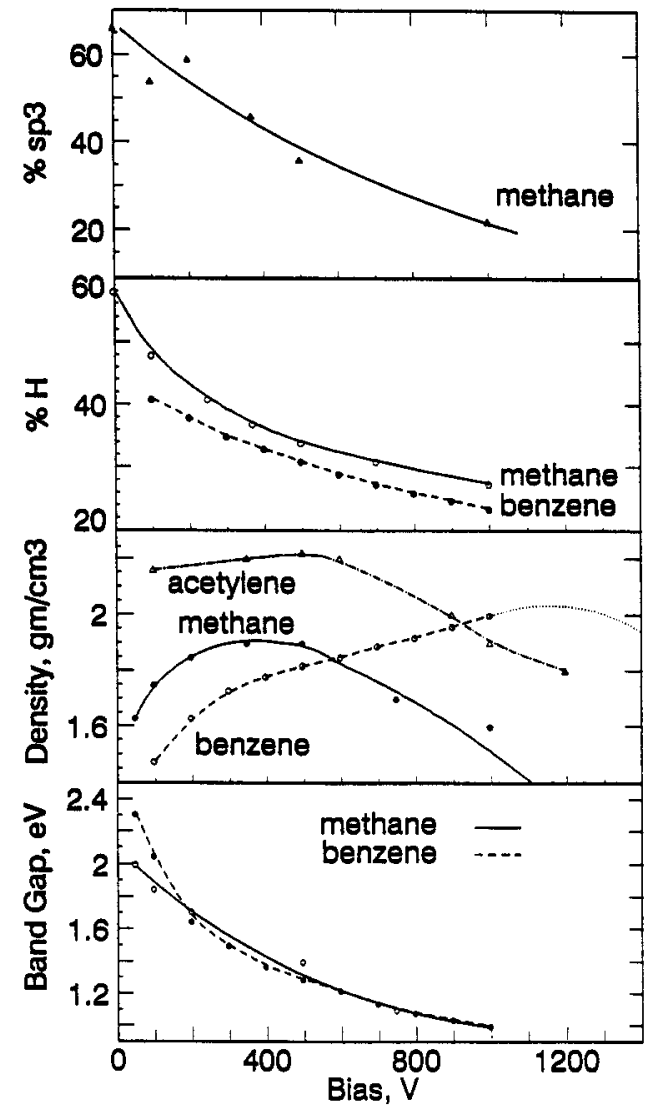

Fig. 5. sp 3 fraction, $\mathrm{H}$ content, density, band gap of PD a-C:H, after $[4,19]$.

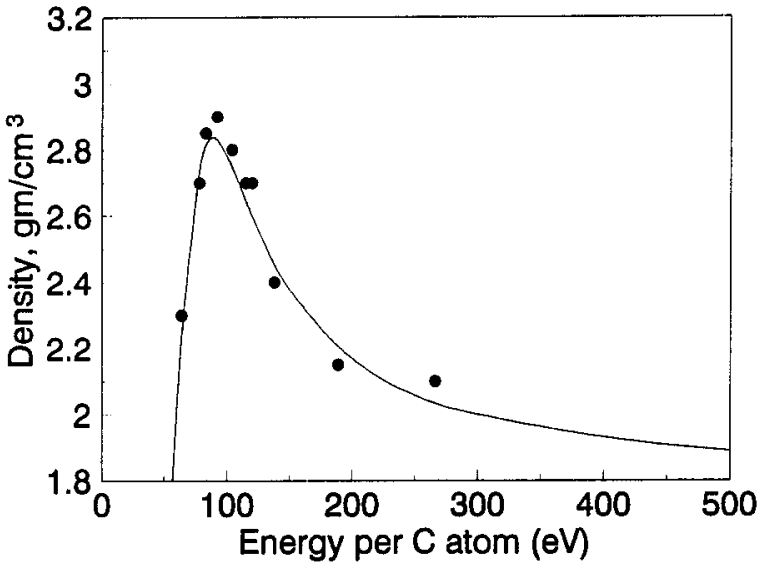

Fig 6. Density vs. ion energy per $\mathrm{C}$ atom of ta-C: $\mathrm{H}$ and fit by eqn (1).

properties of PD a-C: $\mathrm{H}$ depend primarily on bias voltage $V_{b}$ and source gas. $V_{b}$ is a measure of the mean ion energy and at typical operating pressures $(3 \mathrm{~Pa}) \mathrm{E}_{\mathrm{i}} \approx$ $0.4 \mathrm{~V}_{\mathrm{b}}$. Overall, $\mathrm{H}$ content and $\mathrm{sp}^{3}$ fraction decline steadily with $\mathrm{V}_{b}$ (Fig. 5) so that a-C:H has polymeric character at low $V_{b}$, has a maximum density and diamond-like character at intermediate $\mathrm{V}_{\mathrm{b}}$ and has disordered $s^{2}$ bonded character at high $V_{b}[4,19]$. The overall density variation is reminiscent of ta-C, except that the energy scale now depends on source gas. Note that PD a-C: $\mathrm{H}$ (open symbols in Fig. 1) still has sizeable $\mathrm{H}$ and $\mathrm{sp}^{2}$ content which limits its diamond-like character.

Recently, a highly tetrahedral form of a-C:H, 'ta-C:H', was deposited from acetylene using a novel plasma beam source [20]. The electrode configurations allow the powered electrode and plasma to acquire a negative self-bias and a plasma beam exits through a grid into the substrate chamber [21]. The properties of ta-C: $\mathrm{H}$ also depend strongly on ion energy, with the density and $\mathrm{sp}^{3}$ fraction reaching a peak at $200 \mathrm{eV}$ per ion (Fig. 6). The high $\mathrm{sp}^{3}$ fraction (75\% of total C) attained by ta-C:H sets it well above other forms of a-C:H in Fig. 1. The ta- $\mathrm{C}: \mathrm{H}$ also has a high intrinsic compressive stress, and its stress and density each vary linearly with $\mathrm{sp}^{3}$ fraction (Figs. 3,4 ). The high $\mathrm{sp}^{3}$ fraction of this ta-C: $\mathrm{H}$ arises from three factors: the high ionisation of the plasma beam, the mono-energetic character of the ions and the predominance of one ion, $\mathrm{C}_{2} \mathrm{H}_{\mathrm{x}}{ }^{+}$, due to the use of acetylene as a source gas with its simple ionisation pattern.

\section{Deposition Mechanism}

The process creating the metastable, densified phase DLC is called ion subplantation [14,22-24]. At the moderate ion energies of interest, ions they loose their energy mainly by elastic collisions with target nuclei (nuclear stopping) and their range is only a few monolayers. In subplantation, incident ions of sufficient energy penetrate the surface of the growing film, enter interstitial subsurface positions and increase the local density. Penetration occurs by direct entry or knock-on displacement of a surface atom (Fig. 7). Lower energy ions have insufficient energy to penetrate the surface and just stick to the surface to form $\mathrm{sp}^{2} \mathrm{a}-\mathrm{C}$. Higher energy ions penetrate further into the film and increase the density in deeper layers. However, ions need only exceed a certain threshold energy (just less than the displacement threshold) to penetrate the surface, any excess energy rapidly dissipates in a 'thermal spike' [25] during which the excess density can relax by thermally activated diffusion (Fig. 7c). An optimum ion energy occurs where the surface penetration is maximised but relaxation is minimised. Under the energetic conditions of ion bombardment, the local bonding hybridisation adjusts to the local density, becoming more $\mathrm{sp}^{3}$ at high density and more $\mathrm{sp}^{2}$ density at a lower density. The fractional increase in density $\Delta \rho / \rho_{0}$ for a film growing from an incident beam containing a fraction $\phi$ of ions of energy $E_{i}$ is given by 


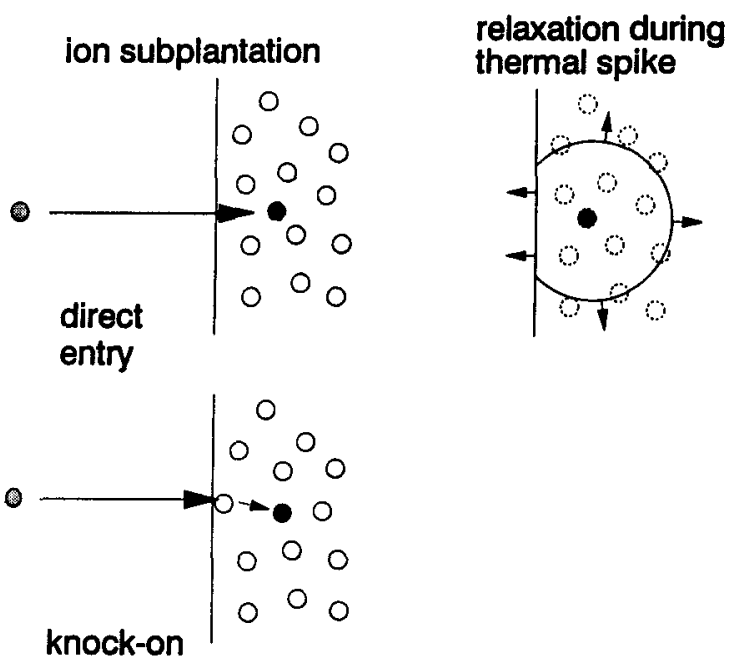

Fig. 7. Two stages of subplantation; ion penetration and density relaxation.

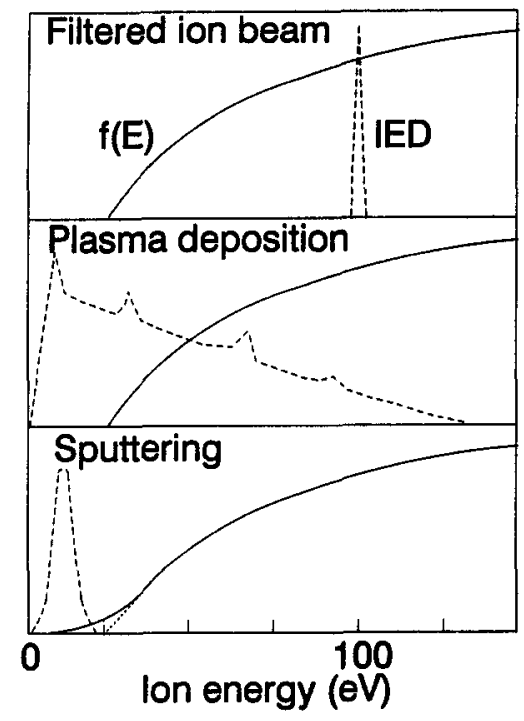

Fig. 8. Schematic penetration probability $(f)$ and ion energy distribution (IED) for filtered ion beam, plasma, and sputter deposition.

$$
\Delta \rho / \rho_{0}=f /\left[(1 / \phi)-f+0.016 p\left(E_{i} / E_{0}\right)^{5 / 3}\right]
$$

where $E_{0}$ is the activation energy of relaxation, $f$ is the penetration fraction and $p$ is a constant of order 1 . The subplantation mechanism describes well the density variation of ta- $\mathrm{C}$ films, as seen in Fig. 2, with $\mathrm{E}_{0}=3.1 \mathrm{eV}, \rho_{0}=2.2 \mathrm{gm} . \mathrm{cm}^{-3}, \mathrm{p}=0.1$ and $\mathrm{f}$ represented empirically by [23] (Fig. 8)

$$
f \approx 1-\exp \left[-\left(\mathrm{E}_{1}-\mathrm{E}_{\mathrm{P}}\right) / \mathrm{E}_{2}\right]
$$

with the penetration threshold $E_{p}=25 \mathrm{eV}$ and a spread factor $E_{2}=57 \mathrm{eV}$ [16].

When the depositing species is a molecular ion such as $\mathrm{C}_{2} \mathrm{H}_{2}{ }^{+}$, the ion fragments on impact at the surface. Its energy partitions, by momentum conservation, roughly equally between two daughter $\mathrm{C}^{+}$ions [18], which then undergo separate subplantations (Fig. 8). The penetration of the daughter ions is characterised by their energy, which is $46 \%$ of the parent ion energy for $\mathrm{C}_{2} \mathrm{H}_{2}{ }^{+}$. The thermal spikes of each daughter ion overlap (Fig. 8b), so the relaxation is characterised by the total energy - the energy of the parent ion. With these adjustments, the subplantation model can also describe the density variation of ta- $\mathrm{C}: \mathrm{H}[20]$, as shown in Fig. 6. The penetration threshold rises markedly to $55 \mathrm{eV}$, as it now includes the energy needed to break the $\mathrm{C}-\mathrm{C}$ bond of $\mathrm{C}_{2} \mathrm{H}_{2}{ }^{+}$on impact. The sharpness of the density dependence in Fig. 6 is the key reason why a monochromatic, single species ion beam is needed to give ta-C:H.

The deposition process is essentially the same in conventional PD a-C:H $[22,23]$. The lower ionisation of the incident beam, the presence of a number of different ions and the broad ion energy spectrum (due to collisions in the plasma sheath, Fig. 9b) convert the sharp density peak of ta-C:H (Fig. 6) into the wider, shallower density peaks seen in Fig. 5. Note that the density of a-C:H prepared from methane, acetylene and benzene reaches a peak at ion energies approximately in the ratio of 1:2:6 (Fig. 5), that is the same energy per daugther $\mathrm{C}$ atom after energy partition [22]. The ion flux also causes dehydrogenation. The incident species have a $\mathrm{H} / \mathrm{C}$ ratio of a least 1 . Ions cause hydrogen loss by preferential sputtering, creating $\mathrm{H}_{2}$ molecules which effuse out of the film. The sputtering cross-section increases with ion energy, causing the $\mathrm{H}$ content to decrease with bias.

The deposition mechanism of sputtered and laser-deposited a-C may also involve subplantation, although further proof is desirable. Sputtering can involve a range of ion energies, the mean is of order $10 \mathrm{eV}$ [8], lower than in ion beam deposition, and lower than the penetration threshold of $\mathrm{C}, 25 \mathrm{eV}$. The penetration 
threshold equals the displacement threshold minus the cohesive energy [23]. In practice, the surface of a-C may be described by a distribution of penetration thresholds, with local low density regions having low thresholds, as shown schematically in Fig. 9(c). The penetration probability, $f$ in (1), would then arise from the convolution of the low energy tail of the penetration distribution and the high energy tail of the ion energy distribution. This would give the relatively low $\mathrm{sp}^{3}$ fractions (but disordered structures) found in sputtered a-C $[7,3]$. A generalisation of such a model could describe many ion beam modification processes, currently given more qualitative descriptions $[10,27]$.

\section{subplantation of molecular ion}
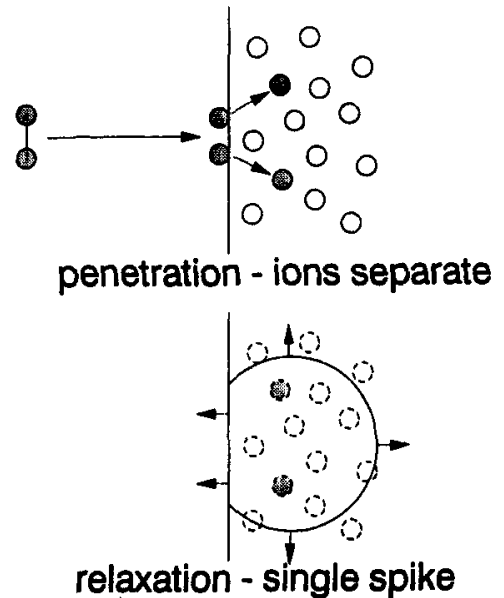

Fig. 9. Subplantation by a molecular ion, with impact fragmentation, separate penetration and a common thermal spike. phases.
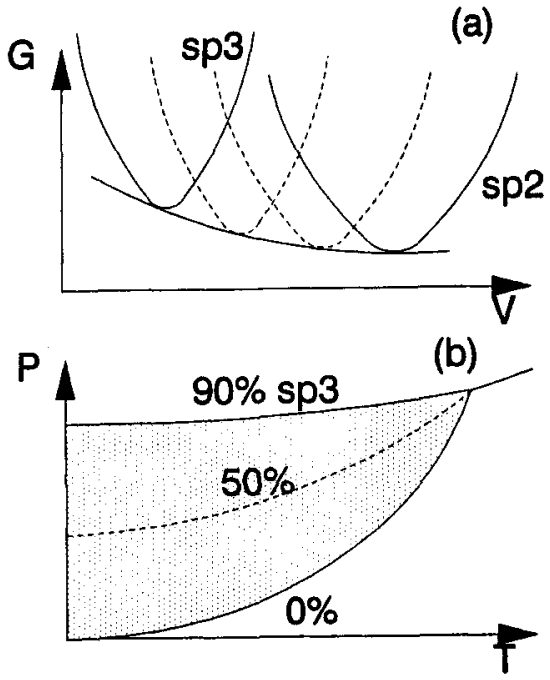

Fig. 10. Energy vs. volume and P-T curves for transitions between a-C phases.

The creation of DLC can also be viewed as a phase transition to a denser phase. The transition from graphite to diamond occurs when the Gibbs free energy of graphite $G(P, T)$ rises above that of diamond. The temperature dependence of the transition pressure between graphite and diamond is known as the Berman-Simon line. McKenzie $[15,27]$ recognised that phases present in deposited thin films could also be described by equilibrium thermodynamics and free energy differences, despite the irreversible nature of the deposition process. The key observation is that most deposition processes create an intrinsic stress, which can be compressive or tensile [28], although it is rarely as large as in DLC. This stress $\sigma$ is biaxial and is equivalent to a hydrostatic pressure of $2 \sigma / 3$ plus a shear of $\sigma / 3$. The hydrostatic component a compressive stress can therefore stabilise a denser metastable phase. McKenzie et al [29] applied these ideas to the deposition of cubic $\mathrm{BN}$ (c-BN). BN, like carbon, has a low density $\mathrm{sp}^{2}$ bonded phase hexagonal $\mathrm{BN}(\mathrm{h}-\mathrm{BN})$ and a denser but less stable $\mathrm{sp}^{3}$ phase, $\mathrm{c}-\mathrm{BN}$. It differs from $\mathrm{C}$ in that its amorphous phases are less prevalent. McKenzie et al [29] found that c-BN was formed when the measured compressive stress exceeded the critical value expected from the known $\Delta \mathrm{G}$ of $\mathrm{c}-\mathrm{BN}$.

In the case of $\mathrm{a}-\mathrm{C}$, the fully $\mathrm{sp}^{3}$ bonded $\mathrm{a}-\mathrm{C}$ is expected to form when the compressive stress exceeds the necessary threshold. Experimentally, the $\mathrm{sp}^{3}$ content of ta-C and ta-C:H varies linearly with stress, and does not display a threshold value (Fig. 4). This suggests that a-C shows a continuous phase transition of increasing $\mathrm{sp}^{3}$ content as shown in Fig. 10(b). This is possible because the random network can accommodate a variable $\mathrm{sp}^{3}$ content, each with its own $\Delta \mathrm{G}$ versus $\mathrm{V}$ curves (Fig. 10a), whereas a sharp transition must occur between the crystalline phases $\mathrm{h}-\mathrm{BN}$ and $\mathrm{c}-\mathrm{BN}$. The difference $\Delta \mathrm{G}$ between $\mathrm{sp}^{2}$ and $\mathrm{sp}^{3} \mathrm{a}-\mathrm{C}$ can be estimated as $\int P \Delta V$ from the difference in density of each form (from Fig. 3) and the hydrostatic pressure equivalent to the total stress of $13 \mathrm{GPa}$ needed to form fully sp ${ }^{3}$ a-C (Fig. 4) to give

$$
\Delta \mathrm{G} \approx 0.10 \mathrm{eV}
$$

This value is larger than that $0.03 \mathrm{eV}$ between graphite and diamond at $0^{\circ} \mathrm{K}$, presumably due to the large distortion energy of the very stiff $\mathrm{sp}^{3}$ bonded random network. 


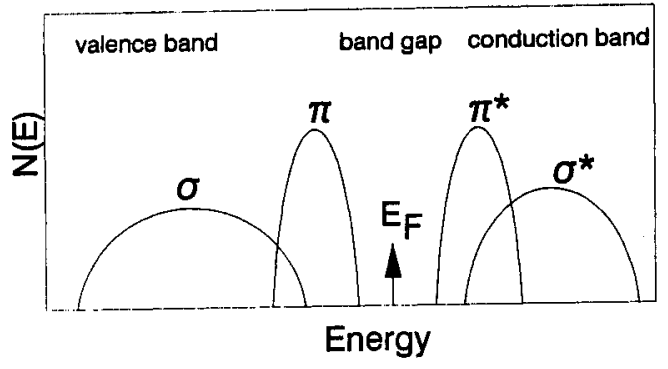

Fig. 11. Schematic density of states of a-C.

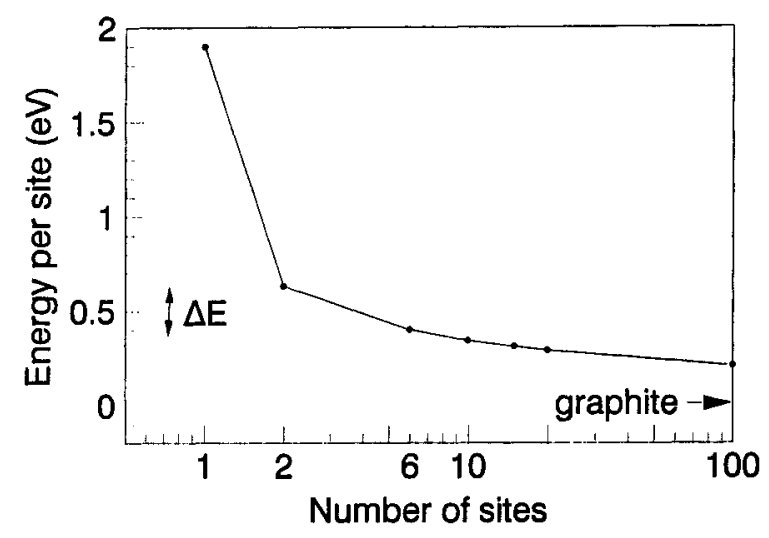

Fig. 12. Variation in total energy with size of $\pi$ bonded clusters, compared to disorder energy $\Delta \mathrm{E}$.

\section{Electronic Structure}

We now consider the atomic structure and electronic structure of a-C and a-C:H. The well known examples of amorphous semiconductors and glasses such as a-Si and a-SiO ${ }_{2}$ are random, $\sigma$-bonded covalent networks. Their chemical bonding is generally the same as in the crystal, with the same coordination number and bond length. Disorder is primarily topological, giving 5- and 7-membered rings in a-Si in addition to the 6membered rings, 'chairs', of crystalline Si. The disorder energy of a-Si roughly equals the elastic energy due to bond length and bond angle distortions. Electronic structure is primarily a function of short range order. Thus, the electronic density of states (DOS) resembles a smoothed version of the crystalline DOS.

$\mathrm{a}-\mathrm{C}$ and a-C:H differ in that they possess both $\sigma$ and $\pi$ bonding. This situation is complex (a) because sp ${ }^{2}$ and $\mathrm{sp}^{3}$ sites have similar energies and (b) because $\pi$ bonding allows more possibilities such as resonance and longer range bonding. Robertson and O'Reilly [30,31] proposed a model for the case of small disorder energy, based on analogies to organic molecules. They noted that the $\sigma$ and $\pi$ states are largely separable, as in the Huckel approximation. The $\sigma$ bonds of $\mathrm{sp}^{3}$ and $\mathrm{sp}^{2}$ sites again form the network's skeleton, and their energetics depend on only their bond lengths and bond angles. The $\pi$ states of $\mathrm{sp}^{2}$ sites in general form a half-filled band. They will gain energy if their arrangement opens up a gap at the Fermi level $E_{\mathrm{F}}$, as this lowers the energy of the occupied states. Many arrangements of $\mathrm{sp}^{2}$ sites will do this. The simplest is a pairing of $\mathrm{sp}^{2}$ sites, as in ethylene. Further energy is gained if $\mathrm{sp}^{2}$ sites form planar 6-fold rings (aromatic stabilisation), and yet further energy if these rings fuse together into aromatic clusters, preferably compact clusters. In this manner, the binding energy of $\pi$ states grows with $\mathrm{sp}^{2}$ cluster size. However, the $\sigma$ binding energy is independent of clustering. Thus, the total binding energy is maximised if $\mathrm{sp}^{2}$ sites and $\mathrm{sp}^{3} \mathrm{sites}$ segregate into a series of $\pi$-bonded clusters embedded in a $\mathrm{sp}^{3}$ bonded matrix.

The $\pi$ states form the band edges and so these states determine the band gap (Fig 11). The band gap of flat aromatic clusters can be calculated in the Huckel approximation to be [30]

$$
E=2 \beta / M^{1 / 2} \approx 6 / M^{1 / 2} e V
$$

where $\mathrm{M}$ is the number of rings in the cluster. This allows $\mathrm{M}$ to be deduced from the observed optical gap. A major factor in favour of aromatic clusters is that they are the smallest configurations to account for the relatively narrow observed optical gaps of $1-2 \mathrm{eV}$ [30-32].

It is now clear that this cluster model is a reasonable model of glassy $\mathrm{C}$ but over-estimates the degree of clustering in interesting forms of a-C such as DLC. Diffraction spectra [33] and molecular dynamics studies [34-36] suggest a much lower degree of ordering of the $\mathrm{sp}^{2}$ sites. The problem is that the disorder potential in interesting forms of $\mathrm{a}-\mathrm{C}$ and $\mathrm{a}-\mathrm{C}: \mathrm{H}$ is quite large, and in particular it is larger than the ordering energies of $\mathrm{sp}^{2}$ sites. Much of our understanding of $\sigma$-bonded networks is gained from a-Si:H. Here, high quality a-Si:H has a disorder potential (deduced from the Urbach slope) of only $0.05 \mathrm{eV}$, much less than a bond energy $2.3 \mathrm{eV}$ or even a 'weak bond' energy of $1 \mathrm{eV}$. In contrast, DLC is purposefully prepared under conditions of ion bombardment, to promote $\mathrm{sp}^{3}$ bonding, which gives a high disorder of typically $0.2-0.3$ 
$\mathrm{eV}$. This is greater than typical ordering energies, which are shown as a function of number of atoms in the cluster in Fig. 12. For example, the aromatic stabilisation of 6 -fold rings is estimated from hydrogenation energies to be $23 \mathrm{kcals}$ or $0.22 \mathrm{eV}$ per site [37] which is similar to the disorder potential. The further energy gain due to fusing of 6-fold rings of $\approx 0.3 \mathrm{eV}$ per site is unlikely to be recovered as it depends so slowly on cluster size. The disorder energy $\Delta \mathrm{E}$ is seen to be similar to the energy gained by clustering. This suggests that clustering will be inhibited by ion bombardment.

A modified model is therefore required to describe the actual case of relatively high disorder. The $\mathrm{sp}^{2}$ sites are likely to form only pairs, single aromatic rings or very small aromatic clusters. However, we must then account for the small size of the gap. Tight-binding calculations on 6-fold $\mathrm{sp}^{2}$ rings have found that strong out-of-plane 'chair' distortions of B symmetry will narrow the gap to about $1.2 \mathrm{eV}$. This would account the optical gaps of most samples of a-C:H but is still too large for sputtered a-C. Odd-membered rings were also considered. Neutral odd-membered rings are not favoured energetically because they have half-filled states near $E_{F}$. However, a combination of a negatively charged 5-fold ring and a positive 7-fold ring, denoted $\mathrm{R}_{5} \cdot-\mathrm{R}_{7}{ }^{+}$, has states at $-1.25 \mathrm{eV}$ and $0.05 \mathrm{eV}$, giving a gap of $1.3 \mathrm{eV}$. Out-of-plane chair distortions of these rings narrows the gap to $0.8 \mathrm{eV}$. It is therefore possible to account for the observed optical gaps in terms of distorted, single $\mathrm{sp}^{2}$ ring structures.

The relative importance of aromatic and olefinic groups still needs more consideration. The original basis for aromatic groups was to account for the observed gap. The presence of aromatic groups is supported by the presence of some shoulder or peak in the Raman spectra at the $1350 \mathrm{~cm}^{-1} \mathrm{D}$ mode of disorder graphitic units in a-C:H [4]. However, high resolution neutron diffraction data of Burke [38] find a C-C sp ${ }^{2}$ bond length of $<1.40 \AA$, suggesting more olefinic than aromatic bonding. The early infra-red spectra of Dischler [39] also showed more olefinic $=\mathrm{CH}$ groups in as deposited a-C:H. Molecular dynamics simulations have not found large aromatic clusters. Galli et al [34] found that the sp ${ }^{2}$ sites of a-C of density $2.2 \mathrm{gm}^{-\mathrm{cm}^{-3}}$ formed thick layers with numerous odd-membered rings. Frauenheim et al [35] find that the $\mathrm{sp}^{2}$ sites of a$\mathrm{C}: \mathrm{H}$ tend to form short chains rather than rings. In contrast, Wang et al $[40,41]$ find mainly aromatic ordering of $\mathrm{sp}^{2}$ sites in simulations of low density a-C and higher density ta-C.

The electronic DOS of a-C(:H) is probed experimentally by a combination of photoemission for the valence band $[42,43]$ and electron energy loss for the conduction band [16]. The $\pi$ states form a knee at top of the valence band and the relative weight of this feature can be used to deduce the $\mathrm{sp}^{2}$ fraction. The sharpness of valence band features shows the transition from polymeric to diamond-like character [42]. The empty $\pi^{*}$ states form a pronounced peak in the C K-edge energy loss spectra at $285 \mathrm{eV}$ below the $\sigma^{*}$ edge at 288 $\mathrm{eV}$. The area of the $\pi^{*}$ peak is a popular, accurate means of deriving the $\mathrm{sp}^{2}$ fraction [16] of a-C(:H) films (infra-red spectra are frequently used, but are inaccurate as they ignore unhydrogenated $\mathrm{C}$ sites [12]).

\section{Mechanical Properties}

The valuable mechanical properties of diamond and DLC such as high Youngs modulus and hardness arise from its strong, directional $\mathrm{sp}^{3}$ bonds. In contrast, the $\sigma$ bonds of graphitic bonding or the $\mathrm{C}-\mathrm{H}$ bonds of hydrocarbon polymers contribute little to the modulus because they do not form a three dimensional network. Thus, the elastic modulus $E$ of the DLC network can be shown to depend only on the mean C-C coordination of the $\mathrm{sp}^{3}$ sites $\mathrm{Z}_{\mathrm{CC}}$ [44-48]. Indeed $E$ varies with $\mathrm{Z}_{\mathrm{CC}}$ as [46]

$$
E=E_{0}\left(\mathrm{Z}_{\mathrm{CC}}-2.4\right)^{1.5}
$$

The hardness $H$ is a measure of the yield strength. Yield in covalent solids occurs by bond breaking, so $H$ can be shown to vary as [47]

$$
H=E / 10
$$

with $E$ given by (5). Equations (5) and (6) were found to give a good description of the modulus and hardness of a-C and a-C:H in terms of the underlying bonding where this is known $[47,48]$.

Acknowledgements. The author is very grateful to $\mathrm{M}$ Weiler and $\mathrm{H}$ Ehrhardt for discussions on their data. 


\section{References}

$1 \quad$ J C Angus, C C Hayman, Science 241877 (1988)

2 K E Spear, this volume

J. Robertson, Prog Solid State Chem 21199 (1991); Surface Coatings Technol 50185 (1992)

P Koidl, C Wild, B Dischler, J Wagner, M Ramsteiner, Mat Sci Forum 5241 (1990)

Y Catherine, in 'Diamond and Diamond-like Carbon', NATO ASI 266B (Plenum, 1991) p193

S Aisenberg, R Chabot, J Appl Phys 422953 (1971)

N Cho, K M Krishnan, D K Vries, M D Rubin, C B Hopper, B Brushan, D B Bogy, J Mater Res 52543 (1990)

$8 \quad$ J J Cuomo, J P Doyle, J Bruley, J C Liu, App Phys Letts 58466 (1991)

9 D L Pappas, K L Saenger, J Bruley, W Krakow, J J Cuomo, J Appl Phys 715675 (1992)

10 J E Sundgren, in 'Diamond and Diamond-like Carbon', NATO ASI 226B (Plenum, 1991) p47

11 R A Street, 'Hydrogenated Amorphous Silicon' (Cambridge University Press, 1991)

12 W Jacob, W Moller, App Phys Lett 631771 (1993)

13 J. Koskinen, J App Phys 632094 (1988)

Y. Lifshitz, S. R. Kasi, J. W. Rabalais, Phys Rev Lett 68620 (1989); Phys Rev B 4110468 (1990)

D R McKenzie, D Muller, B A Pailthrope, Phys Rev Lett 67773 (1991)

P J Fallon, V S Veerasamy, C A Davis, J Robertson, G Amaratunga, W I Milne, J Koskinen, Phys Rev B 484777 (1993)

P H Gaskell, A Saeed, P C Chieux, D R McKenzie, Phys Rev Lett 671286 (1991) W Moller, Appl Phys A 56527 (1993)

M A Tamor, W C Vassell, K R Carduner, App Phys Lett 58592 (1991)

20 M Weiler, S Sattel, K Jung, H Ehrhardt, V S Veerasamy, J Robertson, Appl Phys Lett, to be published (1994)

21 H Oechsner, B Tomcik, Surface Coating Technol 47162 (1991)

22 J Robertson, Phil Trans Roy Soc A 342277 (1993)

J Robertson, Diamond Related Mats 2 (1993) 984; ibid 3361 (1994)

C A Davis, Thin Solid Films 226 (1993) 30

F Seitz, J S Koehler, in 'Solid State Physics' ed. F Seitz and D Turnbull, vol 2 p305 (Academic Press, 1956)

H. Windischmann, J App Phys 621800 (1987)

D R McKenzie, W McFall, W Sainty, C A Davis, R Collins, Diamond Related Mats 2970 (1993)

30 J Robertson, E P O'Reilly, Phys Rev B 352946 (1987)

31 J Robertson, Adv Phys 35317 (1986)

32 J L Bredas, G B Street, J Phys C 18 L651 (1985)

F Li, J S Lannin, Phys Rev Lett 651905 (1991)

G Galli, R M Martin, R Car, M Parinello, Phys Rev Lett 62555 (1989)

Th Frauenheim, P Blaudeck, U Stephan, G Jungnickel, Phys Rev B 484823 (1993)

C H Lee, W R L Lambrecht, B Segall, P C Kelires, Phys Rev B (to be published 1993)

A Streitweiser, C H Heathcock, 'Introduction to Organic Chemistry' (MacMillan, 1985) p565

T Burke, R Newport, W S Howells, K Gilkes, P Gaskell, J Non-Cryst Solids 1641139 (1993)

B Dischler, A Bubenzer, P Koidl, Solid State Commun 48105 (1983)

C Z Wang, K Ho, C T Chan, Phys Rev Lett 70611 (1993)

C Z Wang, K Ho, Phys Rev Lett 711184 (1993)

P Oelhafen, D Ugolini, S Schelz, J Eitle, in 'Diamond and Diamond-like Carbon', NATO ASI, vol 226B (Plenum 1991) p377

43 J Schafer, J Ristein, L Ley, J Non-Cryst Solids 1641123 (1993)

44 J C Phillips J Non-Cryst Solids 34153 (1979)

45 J C Angus, F Jansen, J Vac Sci Technol A 6 (1988) 1778

$46 \quad \mathrm{H} \mathrm{He}, \mathrm{M}$ F Thorpe, Phys Rev Lett 542107 (1985)

47 J Robertson, Phys Rev Lett 68220 (1992)

48 J Robertson, Diamond Related Mats 1397 (1992) 